\title{
Predicción de Fallos Mecánicos en Equipos de Envoltura
}

\author{
Luis F. Rodas y Omar D. Castrillón* \\ Universidad Nacional de Colombia - Sede Manizales - Facultad de Ingeniería y Arquitectura - Departamento de Ingeniería \\ Industrial - Grupo de Innovación y Desarrollo Tecnológico - Bloque Q Campus La Nubia, Manizales, 170001 - Colombia. \\ (e-mail: Ifrodasa@unal.edu.co; odcastrillong@unal.edu.co)
}

* Autor a quien debe ser dirigida la correspondencia.

Recibido Mar. 18, 2019; Aceptado May. 6, 2019; Versión final Jun. 10, 2019, Publicado Dic. 2019

\begin{abstract}
Resumen
El objetivo de este trabajo es el pronóstico de fallos mecánicos en equipos de envoltura partiendo de datos históricos para mejorar la programación de intervenciones preventivas y disminuir el número de correctivas. Para esto se debe recolectar y clasificar datos históricos de tiempos entre fallos, realizar modelos de correlación y a partir de estos resultados definir cuál modelo de pronóstico es mejor, validando los datos mediante pruebas de hipótesis que reduzcan al mínimo la elección de este. Por último, aplicarlo, llegar a resultados y determinar tiempos aptos de programación para mantenimientos preventivos. La metodología empleada condujo al planteamiento de una ecuación que se adapta a las variables que afectan de manera directa el pronóstico del fallo. Se concluyó el impacto positivo de los paros programados en una organización, reduciendo el intervalo de tiempo de intervenciones preventivas y proyectando los tiempos de intervención para el siguiente año.
\end{abstract}

Palabras clave: fallos de equipos; equipos mecánicos; mantenimiento; programación; pronóstico

\section{Prediction of Mechanical Failures in Wrapping Equipment}

\begin{abstract}
The objective of this work is the forecast of mechanical failures in wrapping equipment based on historical data to improve the programming of preventive interventions and reduce the number of corrective maintenances. To do this, it is necessary to collect and classify historical data of time between failures, to perform correlation models and taking these results and define which forecast model is better, validating the data by tests hypotheses that minimize the choice of this. Finally, it was used to obtain results and to determine suitable programming times for preventive maintenance. The methodology used allowed obtaining an equation that is better adapted to the variables that directly affect the failure forecast. It is concluded that a positive impact of the stoppages programmed in an organization was obtained, reducing the time interval of preventive interventions and projecting intervention times for the following year.
\end{abstract}

Keywords: failures of equipment; mechanical equipment; maintenance; programming; forecasting 


\section{INTRODUCCIÓN}

El área de mantenimiento en toda industria moderna se ha convertido en parte esencial del proceso productivo, como indica Stephen (2012) en el entorno comercial competitivo, el tiempo de actividad de las instalaciones no solo es crítico para la continuación del negocio, sino también para mantener una posición de liderazgo en el mercado y disponer en pleno que todos los equipos garantiza un adecuado proceso de fabricación con todo lo que este aspecto demanda, como por ejemplo el aumento productivo y la disminución de los llamados tiempos muertos o improductivos que generan gastos que al final de la cadena de producción merman las utilidades totales de la compañía. Según Espinosa et al., (2008) el área de mantenimiento como función operativa tiene alta relevancia en producción, como relación directa ambas deben funcionar con sinergia para contribuir a la mejora organizacional. Además, de acuerdo con Pinjala et al., (2004) las empresas compiten en el mercado diferenciándose en funciones competitivas como costo, flexibilidad, calidad y disponibilidad.

Es por esto que se hace imperativo garantizar la disponibilidad de los equipos. La ingeniería siempre ha tratado de reducir los tiempos de inactividad y aumentar la disponibilidad de los equipos como dan a entender (Kolte y Dabade, 2017), pero a medida que el factor tiempo y trabajo influye en estos se empiezan a presentar diferentes tipos de deterioro. El natural, relativo al tiempo de uso y el forzoso, aquel que aparece como consecuencia de malas intervenciones, programaciones de mantenimiento obsoletas, materias primas no aptas para la operación de las maquinas entre otros. Lo anterior evidencia que el deterioro forzoso es una variable difícil de controlar ya que se puede presentar por diversos factores.

Con el fin mitigar el efecto negativo en la vida útil de los equipos la industria recurre a los mantenimientos programados, como da entender Pamuk (2015) métodos con actividades que equilibran el mantenimiento contra el impacto de la falla, en otras palabras, son aquellas intervenciones destinadas atacar el problema antes que suceda. Es por lo anterior que hoy en día se ha visto el interés de las empresas por disminuir el número de mantenimientos correctivos, los cuales generan un paro en la producción donde se desconoce el tiempo de la avería y su gravedad. Es así que se presenta la oportunidad de actuar antes de que suceda el problema, haciendo uso de herramientas estadísticas que permitan reducir la cantidad de datos subjetivos recopilados y determinar que variables útiles se pueden usar (Begum y Ahmed, 2015), y matemáticas que permitan prever el tiempo de fallo en un periodo de tiempo evaluado, y al final disminuir los costos de fabricación en los que incurren las empresas por las excesivas intervenciones y paros necesarios para continuar con la producción.

En áreas técnicas como mantenimiento es frecuente que las programaciones e intervenciones preventivas se hagan basadas en la experiencia de las personas encargadas de hacer los mantenimientos, lo que evidentemente crea sesgos en la programación. Si bien es determinante la experiencia del ser humano para conocer su entorno y llegar a soluciones, estas se pueden potenciar haciendo uso de herramientas estadísticas que no son tenidas en cuenta por el afán de alcanzar objetivos organizacionales. Es por lo anterior que la industria siente la necesidad de generar mejores opciones que adecuen sus procesos y lleven en este caso a reducir los mantenimientos correctivos, usando y aplicando de manera correcta y concreta los mantenimientos preventivos.

Uno de los fines de dichos mantenimientos es aumentar el indicador MTBF (Tiempo Medio Entre Fallos). El MTBF es definido por Kolte y Dabade (2017) como el tiempo resultante de restar el tiempo de inactividad desde el tiempo de la operación de la planta, el cual está directamente relacionado con la productividad y eficiencia de una empresa, pilares claves para el objetivo de toda industria que es generar dinero. Así es como la administración le proporciona el valor a la planeación de mantenimiento pues menos fallos implican menos gastos indirectos como reparaciones o menos gastos directos como en producción. Con respecto a una empresa productora de alimentos se ha encontrado que las frecuencias de programación de mantenimientos con el fin de prevenir los fallos mecánicos, para maquinaria industrial de envoltura de caramelo, no son las adecuadas. Primero estas son basadas en la experiencia del técnico o coordinador de mantenimiento y no se rigen por patrones o datos históricos. Segundo, los tiempos actuales difieren en mucho con lo que los datos históricos muestran a simple vista.

Por último, las actividades programadas son generales, es decir no se especifica que componente atacar. Está situación es problemática, cuando las frecuencias son poco reiteradas, o caso contrario, un mantenimiento frecuente impide identificar fallos recurrentes en los equipos. El tiempo exacto de intervención garantiza buenos resultados. Es difícil encontrar el momento óptimo de ajuste ya que el número de mantenimientos correctivos no disminuye y por el mismo deterioro forzoso tiende a aumentar. En promedio por mes las envolvedoras tienen los paros por avería mecánica mostrados en la Tabla 1. Como mencionan (Avilés et al., 2019) la implementación del mantenimiento preventivo y correctivo, se ejecuta de forma regular y llevando a cabo algunos requisitos previos como duración, frecuencia, entre otros. Se observa que en los 
datos anteriores las frecuencias programadas no garantizan la intervención de los componentes, además que lo programado no evidencia lo necesario para dar respuestas a los paros presentados.

Tabla 1: $N^{\circ}$ de averías mensuales por daño mecánico línea envoltura.

\begin{tabular}{|c|c|l|c|}
\hline Equipo & Línea & Frecuencia Preventivo & Recurrencia Mes \\
\hline \multirow{4}{*}{ Envolvedora Rose XXX } & \multirow{4}{*}{ Envoltura } & Ajuste Cuchillas GP & 6 \\
\cline { 3 - 4 } & & Ajuste Cuchillas CC & 1 \\
\cline { 3 - 4 } & & Ajuste Recibidores & 15 \\
\cline { 3 - 4 } & & Ajuste Plegador & 1 \\
\hline
\end{tabular}

Es importante saber que el objetivo del mantenimiento preventivo es reducir los avisos de tipo correctivo, que se muestran como resultado de un paro en un equipo prioritario que frena la producción. A fin de lograr esta meta la industria recurre al TPM (Mantenimiento Productivo Total), la considerada ciencia de las maquinas (Venkatesh, 2005). La empresa objeto de estudio como política organizacional ha empezado a incursionar y ejecutar la metodología TPM. Así, El objetivo principal de este trabajo es desarrollar una metodología consecutiva que permita la obtención de resultados con el fin de pronosticar las averías mecánicas en equipos de envoltura, y establecer el momento en que fallaran para mejorar la programación preventiva y reducir la correctiva. El impacto global generado por una inadecuada planeación se refleja en una producción reducida, lo cual ocasiona pérdida de clientes. Esto muestra una relación con la proyección e intervenciones de equipos. Con el fin de lograr cumplir este objetivo se debe atacar lo relacionado a una inadecuada planeación de los mantenimientos preventivos en donde se encuentran: Que son basados en la experiencia, no hay manejo de datos históricos de averías y los datos históricos pueden presentar sesgo por avisos incorrectos.

Lo anterior hace referencia aquellos avisos que se generan y se cargan a una avería de un componente diferente del que ocurrió. Es por lo anterior que se propuso desarrollar una metodología lógica que pueda tener fundamento a la hora de programar los mantenimientos preventivos aproximando la disponibilidad a un modelo Just in Time (Justo a Tiempo), sistema para empresas que desean lograr la mejor calidad, costo y entrega (Valtanen y Perälä, 2017) mediante el análisis de datos históricos de fallos y hacer uso de modelos de pronósticos para determinar un tiempo de fallo de los componentes críticos en los equipos y programar a partir de estos las intervenciones pertinentes. Los análisis estadísticos de correlación estudian la relación entre dos variables (Asuero et al., 2006). Esta herramienta permite medir la relación entre los datos históricos para seleccionar el modelo de pronóstico. Una vez seleccionado el modelo se procederá a estimar el número de fallas de los periodos siguientes.

Si bien la estadística ha sido usada en diversos trabajos para la predicción de comportamientos muéstrales de datos, este trabajo plantea una metodología que permite facilitar el cálculo a prever, complementado por la ecuación (12) de autoría propia la cual minimiza el error medio. La metodología mostrada a continuación está basada en los métodos de pronósticos de ventas presentadas por Hanke y Wichern, (2010) en su libro "Pronósticos de los Negocios", las cuales fueron adoptadas para el cálculo de fallas en equipos industriales y fueron complementadas con herramientas estadísticas como análisis de correlación y pruebas de hipótesis que permitieran minimizar el error.

\section{MATERIALES Y MÉTODOS}

La metodología aplicada se puede replicar a los diferentes tipos de fallo en equipos homogéneos. Solo es necesario que se sigan cada uno de los pasos estructurados a continuación. A modo de ejemplo se desarrollará para las averías por fallo mecánico de grupo de recibidores. Estos componentes son críticos debido al trabajo continuo y paros poco frecuentes. Los pasos siguientes son consecutivos y tienen como fin la selección del mejor modelo de pronóstico que se ajuste a los datos históricos, dicha metodología sigue los siguientes pasos; clasificación de datos, análisis de correlación, análisis gráfico, selección del modelo de pronóstico.

\section{Paso 1: clasificación de datos}

Lo primero hacer es el análisis de los datos históricos tomando los reportes de los mantenimientos correctivos mensuales por equipo y clasificar los datos de acuerdo a su avería, es decir promediar las averías de las máquinas de la línea por mes y tipo de fallo. Se obtienen los promedios de fallos de cada mes de la línea por grupo recibidores y como paso posterior se realiza una prueba de bondad de ajuste, la cual sirve para determinar el comportamiento de este grupo de datos (Chwialkowski et al., 2016) y conocer a que distribución probabilística se asemejan. 


\section{Paso 2: análisis de correlación}

Con los mismos datos históricos se realiza el análisis de correlación, usada para establecer el grado de asociación entre datos (Asuero et al., 2006), y el cual parte de la ecuación (1) (Hanke y Wichern, 2010a):

$$
\mathrm{r}_{\mathrm{K}}=\frac{\sum_{t=k+1}^{n}\left(y_{t}-\bar{y}\right) *\left(\mathrm{y}_{t-\mathrm{k}}-\overline{\mathrm{y}}\right)}{\sum_{t=1}^{n}\left(y_{t}-\overline{\mathrm{y}}\right)^{2}}
$$

Donde $r_{k}$ es el $r$ de correlación del periodo medido, yt representa el periodo actual $y, y_{t-k}$ es el periodo anterior, $\bar{y}$ es el promedio de los periodos y $\mathrm{n}$ es el tamaño de muestra.

El intervalo de correlación esta entre el rango de -1 y 1 , un coeficiente de correlación 0 indica que no hay relación lineal entre las dos variables estudiadas (Gogtay y Thatte, 2017) o en este caso entre los periodos estudiados. Según Hanke y Wichern (2010c) existe correlación cuando las observaciones en el tiempo están relacionadas. Si los modelos de correlación se usan con series de tiempo, como es este caso de análisis. Estas series se deben estudiar desde un punto de vista moderno o estocástico para dar mayor precisión a su resultado. Se compara el periodo actual con relación al anterior, así para la $n$ de datos que se tenga. Es decir, periodo 2 menos periodo 1 , periodo 3 menos periodo dos...

Una vez determinados los $r$ de correlación, los cuales deben ser igual al tamaño de muestra $(n)$, se determina el porcentaje de error. Para el error es más útil la medida de la calidad de las predicciones hechas por un modelo de regresión (Myttenaere et al., 2015). Se hace uso de las ecuaciones (2) y (3) para el desarrollo de la metodología (Hanke y Wichern, 2010a: p. 68):

$$
\begin{aligned}
& E r_{1}=\frac{1}{\sqrt{n}} \\
& E r_{k}=\frac{\sqrt{1+2\left(\sum_{i=1}^{k} r_{i}^{2}\right)}}{n}
\end{aligned}
$$

En la ecuación (2) se halla el primer error para $r_{1}$, luego se prosigue con la ecuación (3) para los siguientes. Los resultados obtenidos se llevan a la ecuación (4) basada en (Cobo et al., 2014) para hallar los límites de confianza, en conjunto con la distribución obtenida a partir de la prueba de bondad y ajuste. Se debe estimar los parámetros si estos no se conocen mediante el uso de datos que sirvan de muestra. Es esencial ya que los límites dependen de la media, y esta depende de los parámetros (Rao et al., 2011).

$$
\operatorname{Lim}=\mathrm{E}_{(\mathrm{r} 1)} *(\text { Probabilidad })
$$

Los límites de confianza delimitan el rango de confiabilidad de los valores históricos. Una vez obtenidos se procede a graficar el correlograma, en el cual los rk están en función de los límites (Lim) de confiabilidad.

\section{Paso 3: análisis gráfico}

Se analiza el correlograma, de manera que sea visible si hay estacionalidad en los datos. Si es el caso de descarte de estacionalidad se procede con una prueba de hipótesis que genere mayor confiabilidad a la hora de seleccionar el mejor modelo de pronóstico a usar. Se recomienda si es el caso de aceptar o descartar tendencia el estadístico de Ljung-Box, que utilizan la información de variación interna dentro de un objeto de serie temporal a través de muestreo (Lee y Park, 2016). La prueba de hipótesis Ljung-Box es descrita por las ecuaciones (6) (Hanke y Wichern, 2010a), y (5), (7) basadas en (Giraldo, 2006).

$$
\begin{aligned}
& Q=t \sum_{j=i}^{L}\left(r_{j}^{2}\right) \\
& r_{k}=\frac{\sum_{t=k+1}\left(e_{t}{ }^{*} e(t-k)\right)}{\sum_{t=1} e_{t}^{2}} \\
& Q_{L B}=n(n+2) * \sum_{j=1}^{L} \frac{r_{j}^{2}}{t-j}
\end{aligned}
$$


Las ecuaciones (5), (6) y (7) están relacionadas de manera directa con los $r$ de correlación hallada en el paso 2. Donde $Q$ representa la prueba de hipótesis y QடB la prueba de hipótesis por estadístico Ljung-Box. Una vez hallados los valores del Q prueba de hipótesis: $\mathrm{H}_{\circ} \alpha=0$; Hay tendencia en los datos; $H_{\circ} \alpha \neq 0$; No hay tendencia en los datos. Se debe cumplir que $X^{2} \geq Q\left\llcorner B\right.$, donde $X^{2}$ representa la distribución Chi Cuadrada.

\section{Paso 4: selección del modelo de pronóstico}

En este paso ya se debe saber con certeza qué modelo de pronóstico aplicar. Se puede encontrar modelos de pronósticos cualitativos en los cuales los métodos reciben poca atención ya que son solo aplicaciones estándar (Meimand et al., 2012) y métodos cuantitativos, siendo de interés para este estudio los que se basan en números, además según Vitri (2014) el papel del pronóstico de demanda cambia dependiendo de la posición que la cadena de abastecimiento ocupa en la empresa. Para determinar el modelo del pronóstico se partirá de un modelo de correlación serial conocido como regresión con diferencias que permite analizar series de tiempo para pronósticos en la cual el error del periodo actual está directamente relacionado con el error del periodo anterior y se compone de las siguientes ecuaciones (Hanke y Wichern, 2010c: p. 340):

$$
\begin{aligned}
& y_{t}=B_{0}+B_{1} x_{t}+£_{t} \\
& £_{t}=\rho £_{t-1}+v_{t}
\end{aligned}
$$

Donde $\rho=$ Correlación entre dos errores consecutivos, $v_{t}=$ Error aleatorio, $v_{t}=£_{t}$ cuando $\rho=0$. La ecuación (8) muestra el efecto de la correlación serial. La ecuación (9) muestra que el error del término anterior afecta el siguiente. $-1 \leq \rho \geq 1$.

Lo anterior indica la fortaleza de la correlación, si es 0 entonces no existe correlación serial y los términos de error son independientes. Conociendo el tipo de modelo se determina que técnica es mejor para el proceso. Se optó por escoger un modelo de suavización exponencial simple, Ostertagova y Ostertag (2012) dan a entender que el modelo de suavización exponencial simple SES es usualmente basado en la premisa de que el nivel de las series de tiempo debe fluctuar alrededor de un nivel constante o cambiar lentamente sobre el tiempo. Este modelo es expresado en ecuación (10) (Hanke y Wichern, 2010b: p. 120):

$$
y_{t+1}=y_{t}+a\left(Y_{t}-\bar{Y}_{t}\right)
$$

Donde se relaciona el periodo anterior con el actual, y $\alpha$ es un valor con intervalo entre $(0,1)$. Además, según Ravinder (2013) las constantes de suavización determinan la sensibilidad de los pronósticos a los cambios. Grandes valores de a hacen que estos respondan mejor a niveles más recientes, mientras que los valores más pequeños tienen un efecto de amortiguamiento. Por ejemplo, un a de 1 que es el mayor hará que el pronóstico sea exactamente igual al del último periodo. La ecuación (11) (Morales, 2001: p. 586), muestra el error medio del pronóstico. (Chen et al., 2017) afirma que una buena medida de precisión debe proporcionar un resumen informativo y claro de la distribución de los errores.

$$
\operatorname{PEMA}=\frac{\Sigma e_{t}}{(\mathrm{n}) *\left(\mathrm{y}_{\mathrm{t}}\right)}
$$

Además, la correcta aplicación de nuevos modelos como precisan Liu et al., (2018) como algoritmos de predicción a mediano y largo plazo que están basados en modelos de suavización exponencial pueden llevar a la reducción del error en el pronóstico en un $35,37 \%$. Siempre el pronóstico busca minimizar el porcentaje de error para dar mejor fiabilidad y confiabilidad a los procesos que desencadene.

\section{RESULTADOS}

Los resultados a continuación expuestos son efecto de la metodología desarrollada en el apartado anterior, teniendo como fuente los datos históricos de avería por grupo recibidores de la empresa objeto de estudio:

\section{Paso 1: clasificación de datos}

Se clasificaron los datos históricos y se dividieron según el tipo de avería, como se ha aclarado con anterioridad los resultados solo se mostrarán para el fallo por grupo recibidores. Posterior a esto se promediaron todas las máquinas de la línea. 
La Tabla 2 muestra los resultados y promedios de falla por grupo recibidores, siendo estos datos los que finalmente se utilizaran para el procedimiento. Una vez obtenido los datos promedio se realiza una prueba de bondad y ajuste. A fin de facilitar la operación se realizan pruebas de bondad y ajuste rápido mediante el complemento Stat-Fit del software de simulación Promodel el cual es usado para simular procesos en su mayoría industriales y permite el análisis de distribuciones estadísticas a partir de un conjunto de datos.

Tabla 2: Promedio datos avería por grupo recibidores.

\begin{tabular}{|c|l|c|}
\hline Periodo & \multicolumn{1}{|c|}{ Mes } & Fallas/Mes \\
\hline & & \\
\hline 1 & Junio 2016 & 13,3 \\
\hline 2 & Julio 2016 & 7,5 \\
\hline 3 & Agosto 2016 & 15,0 \\
\hline 4 & Septiembre 2016 & 12,9 \\
\hline 5 & Octubre 2016 & 9,0 \\
\hline 6 & Noviembre 2016 & 14,7 \\
\hline 7 & Diciembre 2016 & 13,7 \\
\hline 8 & Enero 2017 & 13,8 \\
\hline 9 & Febrero 2017 & 14,4 \\
\hline 10 & Marzo 2017 & 16,0 \\
\hline 11 & Abril 2017 & 11,8 \\
\hline 12 & Mayo 2017 & 13,9 \\
\hline 13 & Junio 2017 & 15,7 \\
\hline 14 & Julio 2017 & 10,2 \\
\hline
\end{tabular}

\begin{tabular}{|c|l|c|}
\hline Periodo & \multicolumn{1}{|c|}{ Mes } & Fallas/Mes \\
\hline & & \\
\hline 15 & Agosto 2017 & 10,3 \\
\hline 16 & Septiembre 2017 & 17,5 \\
\hline 17 & Octubre 2017 & 20,1 \\
\hline 18 & Noviembre 2017 & 13,8 \\
\hline 19 & Diciembre 2017 & 14,8 \\
\hline 20 & Enero 2018 & 16,0 \\
\hline 21 & Febrero 2018 & 13,1 \\
\hline 22 & Marzo 2018 & 8,5 \\
\hline 23 & Abril 2018 & 8,7 \\
\hline 24 & Mayo 2018 & 10,0 \\
\hline 25 & Junio 2018 & 12,3 \\
\hline 26 & Julio 2018 & 12,8 \\
\hline 27 & Agosto 2018 & 14,0 \\
\hline
\end{tabular}

Los resultados mostrados en la Tabla 3 son producto del análisis de bondad y ajuste efectuado por medio del complemento Stat-Fit (ajuste rápido) del software Promodel. En la Tabla 3 se observa la distribución a la cual los datos mejor se adaptan, en este caso una distribución estadística normal. Se realiza las pruebas de bondad y ajuste de acuerdo a esta distribución, dando un alfa de 0,05 que equivale a un $95 \%$ de confiabilidad. Los detalles de estos resultados, donde se observa que no existe un rechazo de las pruebas, son ilustrados a continuación:

Tabla 3: Pruebas de bondad y ajuste para distribución aceptada, resultado por ajuste rápido.

\begin{tabular}{|l|l|l|}
\hline \multicolumn{3}{|c|}{ Auto fit of distributions } \\
\hline Distribution & Rank & Acceptance \\
\hline Uniform $(7,1.63,0.702)$ & 100 & reject \\
\hline Lognormal $(7,20.1)$ & 52,4 & do not reject \\
\hline Exponential $(7,6.11)$ & 2,88 & reject \\
\hline Lognormal & \\
\hline minimun & 7 (fixed) & \\
\hline maximun & 20,12 & \\
\hline Kolmogorov-Smirnov & & Anderson-Darling \\
\hline Data points 12 & & Data points 26 \\
\hline Ks stat 0,236 & & Ad stat 2,12 \\
\hline Alpha 0,05 & Alpha 0,05 \\
\hline Ks stat $(27,0.05)$ 0,254 & & Ks stat $(27,0.05) 2,49$ \\
\hline p-value 0,0829 & & p-value 0,0767 \\
\hline DO NOT REJECT & & DO NOT REJECT \\
\hline
\end{tabular}

\section{Paso 2: análisis de correlación}

Se procede a usar las ecuaciones (1), (2) y (3) para hallar los $r$ de correlación por cada periodo. Mediante la ecuación (4) se halla los límites de confianza a partir de los parámetros que definen la distribución normal (Media, Desv. Estándar): Media =13,11; Desviación Estándar =2,93; Distribución Normal $(0,05)=8,29$. Los resultados obtenidos se expresan en la Tabla 4. Como resultado de esta se obtiene la Fig. 1. 
Tabla 4: Datos de correlación y límites superiores e inferiores de confianza, por periodo.

\begin{tabular}{|c|c|c|c|}
\hline Periodo & Correlación & Límites Positivos & Límites Negativos \\
\hline 1 & 0,2359 & 1,5954 & $-1,5954$ \\
\hline 2 & $-0,1238$ & 0,3237 & $-0,3237$ \\
\hline 3 & 0,1566 & 0,3281 & $-0,3281$ \\
\hline 4 & $-0,0264$ & 0,3351 & $-0,3351$ \\
\hline 5 & $-0,3807$ & 0,3353 & $-0,3353$ \\
\hline 6 & $-0,2469$ & 0,3738 & $-0,3738$ \\
\hline 7 & 0,0162 & 0,3888 & $-0,3888$ \\
\hline 8 & $-0,0608$ & 0,3889 & $-0,3889$ \\
\hline 9 & 0,1329 & 0,3898 & $-0,3898$ \\
\hline 10 & 0,1612 & 0,3941 & $-0,3941$ \\
\hline 11 & $-0,0841$ & 0,4002 & $-0,4002$ \\
\hline 12 & $-0,1238$ & 0,4019 & $-0,4019$ \\
\hline 13 & 0,0228 & 0,4055 & $-0,4055$ \\
\hline 14 & $-0,1323$ & 0,4056 & $-0,4056$ \\
\hline 15 & $-0,2724$ & 0,4097 & $-0,4097$ \\
\hline 16 & $-0,0696$ & 0,4264 & $-0,4264$ \\
\hline 17 & 0,0326 & 0,4275 & $-0,4275$ \\
\hline 18 & $-0,0089$ & 0,4277 & $-0,4277$ \\
\hline 19 & 0,0193 & 0,4277 & $-0,4277$ \\
\hline 20 & 0,0967 & 0,4278 & $-0,4278$ \\
\hline 21 & 0,0943 & 0,4298 & $-0,4298$ \\
\hline 22 & 0,0527 & 0,4318 & $-0,4318$ \\
\hline 23 & 0,0146 & 0,4324 & $-0,4324$ \\
\hline 24 & 0,0150 & 0,4324 & $-0,4324$ \\
\hline 25 & $-0,0217$ & 0,4325 & $-0,4325$ \\
\hline 26 & 0,0006 & 0,4326 & $-0,4326$ \\
\hline
\end{tabular}

Paso 3: análisis gráfico

En la Fig. 1 se denota el alto componente aleatorio, y unos pocos periodos con estacionalidad la cual es despreciable comparada con la tendencia en datos seguidos (ver barras fig.1), que es tanto ascendente como descendente en algunos periodos continuos. En la Tabla 5 se muestran los resultados obtenidos a través de la prueba de hipótesis Ljung-Box. La prueba de hipótesis acepta la hipótesis nula y se concluye que no hay tendencia, los datos presentan un comportamiento aleatorio.

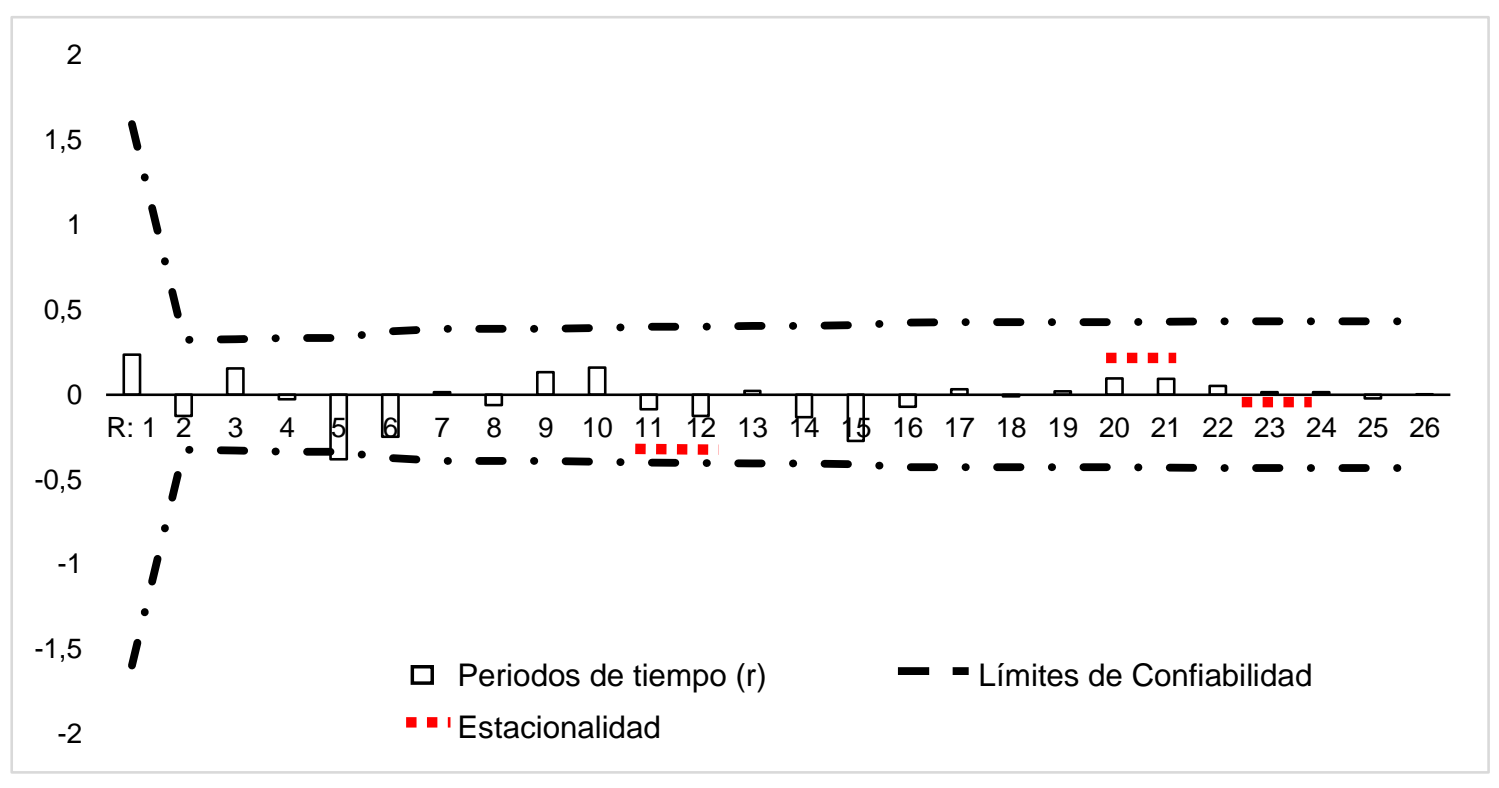

Fig. 1: Correlograma Fallos Grupo Recibidores. 
Tabla 5: Comparativo Distr, Chi Cuadrada, Estadístico Ljung-Box.

\begin{tabular}{|c|c|c|}
\hline Periodo & Ljung-Box & Chi-Cuadrado \\
\hline r1 & 3,3528 & 3,8415 \\
\hline r2 & 2,1566 & 5,9915 \\
\hline r3 & 2,4765 & 7,8148 \\
\hline r4 & 1,7001 & 9,4878 \\
\hline r6 & 6,8339 & 11,0705 \\
\hline r7 & 3,9502 & 12,5916 \\
\hline r8 & 1,6867 & 14,0671 \\
\hline r9 & 1,8290 & 15,5073 \\
\hline 10 & 2,4451 & 16,9190 \\
\hline 11 & 2,8727 & 18,3070 \\
\hline 12 & 2,0223 & 19,6751 \\
\hline r13 & 1,7055 & 21,0261 \\
\hline r14 & 2,7313 & 22,3620 \\
\hline r15 & 6,5175 & 23,6848 \\
\hline r16 & 2,0213 & 24,9958 \\
\hline r17 & 1,7598 & 26,2962 \\
\hline r18 & 1,6834 & 27,5871 \\
\hline 19 & 1,7128 & 28,8693 \\
\hline 20 & 2,7231 & 30,1435 \\
\hline 21 & 2,8358 & 31,4104 \\
\hline r22 & 2,1120 & 32,6706 \\
\hline r23 & 1,7179 & 33,9244 \\
\hline r24 & 1,7354 & 35,1725 \\
\hline r25 & 1,8613 & 36,4150 \\
\hline 26 & 1,6767 & 38,6525 \\
\hline & & \\
\hline & & 3851 \\
\hline
\end{tabular}

\section{Paso 4: selección del modelo de pronóstico}

Las pruebas dieron como resultado que los datos se ajustan a modelos de pronóstico aleatorio. El modelo aleatorio aplicado es la suavización exponencial simple ya que es el que mejor se adapta al proceso y el que menor porcentaje de error arroja debido al factor de ponderación que permite una relación directa para el resultado del pronóstico. Se opta por un modelo de suavización exponencial simple, que permita buscar una relación con el indicador MTBF. Se desarrolló un nuevo paso para incorporar las variables que afectan el proceso y se pueden incluir dentro del factor de ponderación $\alpha$. Como nos dan a entender Chusyairi y Ramada (2018), el modelo de suavización exponencial simple es un método de excelente rendimiento, de fuerte adaptabilidad y de fácil análisis de datos.

Determinación del factor de ponderación

Para el caso de estudio se encuentra que hay necesidad de relacionar el modelo predictivo con el indicador MTBF. Basado en los datos y variables implícitas que afectan el resultado final del pronóstico, se crea una ecuación que supla las necesidades y se adapte solo a las variables que afecten el proceso. La ecuación (12), se desarrolló para mostrar relación con el indicador de tiempos entre fallos (MTBF) y las variables que afectan de manera directa e indirecta el número de fallos por componente de los equipos en un tiempo medido:

$$
\alpha=\frac{t^{*}(\operatorname{Ln}(n))}{\operatorname{MTBF}(n)}(k)
$$

Dónde: $\alpha=$ Factor de Ponderación, $t=$ Unidad de tiempo. Congruente con el indicador MTBF, $\mathrm{n}=$ Tamaño de muestra, $\mathrm{MTBF}=$ Indicador de tiempo entre fallos. Tiempo de buen funcionamiento, $\mathrm{K}=$ Constante de deterioro forzoso. Se asocian al factor de ponderación $\alpha$ las siguientes variables: $t$ que es directamente proporcional y debe estar en la misma unidad de tiempo que el MTBF, n que es Inversamente proporcional, es decir que entre mayor sea el tamaño de muestra menor será $\alpha$, constante de deterioro forzoso $\mathrm{k}$, que es directamente proporcional, es decir a mayor $\mathrm{k}$ de deterioro mayor será $\alpha$. Se propone una función logarítmica de manera que $n$ siempre sea menor en el numerador y garantice una relación proporcional y estable a la ecuación. Según Villa (2014); Hanke y Wichern (2010c) una función logarítmica aplicada a la oferta y demanda permite calcular con más exactitud la variable de interés, disminuyendo el porcentaje de error. 
Además, las constantes de deterioro forzoso se clasificaron de acuerdo a los siguientes valores numéricos: $\mathrm{k}=1$. Cuando los datos históricos muestran un comportamiento estable. Aplica para modelos de pronósticos estacionarios. $\mathrm{k}=3$. Cuando los datos históricos muestran un comportamiento aleatorio. Aplica para modelos de pronósticos aleatorios. $k=5$. Cuando los datos históricos muestran un comportamiento creciente. Aplica para modelos de pronósticos de tendencia. Una vez obtenidos los resultados, se obtendrán las frecuencias para los mantenimientos por mes. A partir de la ecuación (10) se halla el alfa de la ecuación (12). Tomando el tiempo como 480 min que debe funcionar la máquina por turno, y como ejemplo se toma el MTBF como 450 min que funciono la máquina y un $\mathrm{k}$ de deterioro forzoso igual a 3 , es decir adaptado a una muestra de datos históricos aleatorios se obtiene el resultado mostrado en la ecuación (13):

$$
\alpha=\frac{480 \min *(\operatorname{Ln}(27))}{\operatorname{MTBF}(27)}(3)=0,39
$$

Se determina el pronóstico para cada periodo del año siguiente a partir del alfa y la ecuación (12). Estos resultados se evidencian en la Tabla 6.

Tabla 6: Datos pronósticos de fallos por mes para años siguientes.

\begin{tabular}{|l|c|c|c|c|c|c|c|}
\hline Periodo & Año 2015 & Año 2016 & Año 2017 & Año 2018 & Año 2019 & Año 2020 & Año 2021 \\
\hline & & & & & & & \\
\hline Enero & & 13,83 & 16,03 & 15,17 & 15,51 & 15,38 & 15,43 \\
\hline Febrero & & 14,43 & 13,10 & 13,62 & 13,41 & 13,49 & 13,46 \\
\hline Marzo & & 16,05 & 8,45 & 11,42 & 10,26 & 10,71 & 10,54 \\
\hline Abril & & 11,83 & 8,69 & 9,92 & 9,44 & 9,63 & 9,55 \\
\hline Mayo & & 13,86 & 10,00 & 11,51 & 10,92 & 11,15 & 11,06 \\
\hline Junio & 13,28 & 15,71 & 12,30 & 13,63 & 13,11 & 13,32 & 13,24 \\
\hline Julio & 7,50 & 10,24 & 12,78 & 11,79 & 12,17 & 12,02 & 12,08 \\
\hline Agosto & 15,00 & 10,29 & 13,97 & 12,53 & 13,09 & 12,87 & 12,96 \\
\hline Septiembre & 12,94 & 17,50 & 15,72 & 16,41 & 16,14 & 16,25 & 16,21 \\
\hline Octubre & 9,05 & 20,12 & 15,79 & 17,48 & 16,82 & 17,08 & 16,98 \\
\hline Noviembre & 14,74 & 13,81 & 14,17 & 14,03 & 14,09 & 14,06 & 14,07 \\
\hline Diciembre & 13,71 & 14,84 & 14,40 & 14,57 & 14,51 & 14,53 & 14,52 \\
\hline
\end{tabular}

El error medio fue de 1,5\%. Los resultados anteriores se dividen entre el número de días del mes en que la máquina está programada y se multiplica por el número de turno que esta trabaja. En la Tabla 7 se aprecian los resultados y las nuevas frecuencias de mantenimientos preventivos por mes para el grupo de recibidores en la maquinaria objeto de análisis. Por ejemplo, de acuerdo a la información presente en la Tabla 7 en el mes de enero de 2019 se debe intervenir la máquina en el grupo recibidores en promedio cada 3 días de funcionamiento (1117 $\mathrm{min}$ ).

Tabla 7: Frecuencias por minuto para intervención en máquinas por mes.

\begin{tabular}{|l|c|c|c|}
\hline \multirow{2}{*}{ Periodo } & \multicolumn{3}{|c|}{ Frecuencia Ejecución Plan Preventivo (min) } \\
\cline { 2 - 4 } & Año 2019 & Año 2020 & Año 2021 \\
\hline Enero & 1117 & 1108 & 1111 \\
\hline Febrero & 966 & 972 & 970 \\
\hline Marzo & 739 & 772 & 759 \\
\hline Abril & 680 & 694 & 688 \\
\hline Mayo & 787 & 803 & 797 \\
\hline Junio & 945 & 959 & 954 \\
\hline Julio & 877 & 866 & 870 \\
\hline Agosto & 943 & 927 & 933 \\
\hline Septiembre & 1163 & 1170 & 1167 \\
\hline Octubre & 1212 & 1230 & 1223 \\
\hline Noviembre & 1015 & 1013 & 1014 \\
\hline Diciembre & 1045 & 1047 & 1046 \\
\hline
\end{tabular}




\section{DISCUSION}

Conocer el tiempo de falla permite disponer de manera óptima el mantenimiento preventivo. En el artículo titulado "Pronosticar el tiempo de fallo de las máquinas usando un algoritmo neuro-genético híbrido" del autor Paithankar (2016), se encuentra un trabajo con un objetivo similar, predecir fallos en equipos, pero con un enfoque teórico y práctico diferente. Ambos trabajos difieren en la metodología usada, si bien el presente escrito busca adoptar modelos de pronósticos utilizados en ventas, el artículo en mención desarrollo un método propio de algoritmo genético con sus nodos de entrada, nodos ocultos y algoritmos de aprendizaje.

Es de reconocer que el método empleado es heurístico y el utilizado en el desarrollo de este trabajo es estocástico, sabiendo la exactitud y el alcance que tiene el uno sobre el otro. Como nombra el autor métodos tradicionales han dejado su efectividad ejemplo claro, cadenas de markov y análisis de árbol de fallas entre otros. Para las primeras se ha demostrado que muchos de los sistemas necesitan ser modelados en otro orden, como mencionan Prasad y Prasad (2014) tienen ciertas limitaciones a la hora de suponer que una distribución estadística específica, se ajusta a los datos históricos de fallos, y tiene lógica pues métodos básicos como estos no suplen una necesidad adecuada para los problemas planteados. Como mencionan (Sánchez, G. et. al, 2004) el desarrollo de redes crecientes que agregan nodos de aprendizaje en forma sistemática representan una alternativa más atractiva.

Además, las redes neuronales aplicadas a pronósticos han probado su efectividad y confiabilidad, demostrando que pueden aproximarse a cualquier función continua. Datos financieros han sido pronosticados a partir de redes neuronales (Tealab, 2018) entre otras áreas, haciendo a un lado métodos más sencillos, por lo que el futuro de los métodos predictivos debe cambiar y enfocarse a modelos más fuertes que se adapten a las necesidades actuales, y un aspecto importante del artículo citado es que su teoría fue medible y se validó en campo, demostrando resultados satisfactorios en la predicción de fallos. Las redes neuronales ofrecen una poderosa herramienta para evaluar datos y parámetros de las máquinas que pueden aprender de los datos de proceso de la simulación de fallas (Krenek et al., 2016).

Hay técnicas que en la actualidad son relevantes para predecir fallos inminentes de los sistemas, técnicas para la clasificación de variables en el tiempo, de series de tiempo multivariadas (Westholm, 2018), pasar de pensar en un sistema lineal a uno interconectado, acompañando la adopción de los avances recientes en los métodos de análisis de big data que ayudarán a desarrollar una estrategia efectiva de mantenimiento preventivo (Sujata et al., 2018). Por ejemplo ya se están desarrollando máquinas inteligentes que gracias al despliegue de sensores de medición permiten comunicar el fallo en tiempo real (Exner, 2018). Es por lo anterior que modelos complejos deben adaptarse a entornos complejos y los simples a casos de fácil aplicación, dejando en claro que el presente y futuro de las predicciones en todo entorno empresarial deben migrar a modelos heurísticos métodos que puedan generar simplicidad y flexibilidad en los algoritmos, manejando una gran cantidad de restricciones (Dawid et al., 2017).

Los modelos de pronósticos en la industria han tenido acogida referenciados a planes y proyecciones de venta. Este trabajo intenta dar una aproximación y aumentar la disponibilidad de equipos y maquinarias que componen el entorno de una empresa. Para tal fin se asocia los modelos de pronósticos con el objetivo de predecir fallos dando uso a los históricos de paros en las máquinas. Los porcentajes de errores que estos arrojan pueden variar debido al sesgo de la información, y al tamaño de la muestra, pues más datos generan un mejor resultado. Siempre habrá un error debido al componente aleatorio de las muestras, el objetivo es minimizarlo, en este caso afianzando el coeficiente alfa que afecta directamente al pronóstico. El cambio en la programación de los planes preventivos busca por sobre todo reducir los costos indirectos que afectan producción y aumentar la disponibilidad de las maquinas, aportando a la metodología just in time. La ecuación (12) que surgió producto de esta investigación aún puede presentar sesgos, pero se considera que es una buena aproximación para el objetivo de este trabajo.

\section{CONCLUSIONES}

De acuerdo al trabajo presentado y a los resultados obtenidos, se pueden plantear las siguientes conclusiones principales:

1.- Los resultados ayudan a proyectar en un tiempo indicado las intervenciones para el componente objeto de estudio (Grupo Recibidores), con el fin de disminuir los paros no programados.

2.- Se logró disminuir los tiempos para las intervenciones preventivas, permitiendo programar los mantenimientos de acuerdo a las necesidades de los equipos. 
3.- Se logró adaptar la ecuación (12) de manera que las variables que afectan directamente el fallo del equipo están relacionadas al pronóstico y el error medio varía de acuerdo al resultado del coeficiente hallado.

\section{AGRADECIMIENTOS}

Se agradece la colaboración prestada a la Universidad Nacional de Colombia Sede Manizales y al Departamento de Ingeniería Industrial.

\section{REFERENCIAS}

Asuero, A., A. Sayago y A. González, The Correlation Coefficient: An Overview, Doi: 10.1080/10408340500526766, Critical Reviews in Analytical Chemistry, 36, 41-59 (2006)

Avilés, M., K. Brito, y C. Mendoza, Análisis Bibliográfico sobre el Impacto del Mantenimiento Preventivo y Correctivo en el Sector Público, http://repositorio.unemi.edu.ec/handle/123456789/4450, Tomado de: Universidad Estatal de Milagros, (2019)

Chen, C., J. Twycross y J. Garibaldi, A new accuracy measure based on bounded relative error for time series forecasting, Doi: 10.1371/journal.pone.0174202, Plos One, 12(3), (2017)

Chwialkowski, K., H. Strathmann y A. Gretton, A Kernel Test of Goodness of Fit, arXiv:1602.02964, London, Cornell University, JMLR: Workshop and Conference Proceedings (2016)

Chusyairi, A. y P. Ramadar, The Use of Exponential Smoothing Method To Predict Missing Service E-Report, Doi:

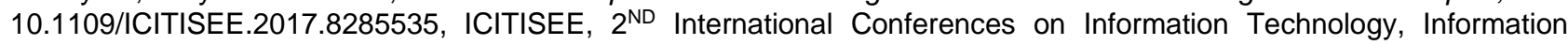
Systems and Electrical Engineering, 39-44 (2018)

Cobo, E., B. Kostov, y otros tres autores, Capítulo 8: Intervalos de Confianza, Bioestadística para no Estadísticos, Masson, 24-25, Barcelona, España (2014)

Dawid, R., D. McMillan y M. Revie, Heuristic algorithm for the problem of vessel routing optimisation for offshore wind farms, Doi: 10.1049/joe.2017.0511, The Journal of Engineering, $2^{\mathrm{ND}}$ International Conferences on Information Technology, Information Systems and Electrical Engineering (13), (2017)

De Myttenaere, A., B. Golden, y otros 2 autores, Using the Mean Absolute Percentage Error for Regression Models, Doi: 10.1016/j.neucom.2015.12.114, HAL archieves-ouvertes, 192, 38-48 (2015)

Exner, C, Implementation of a Predictive Maintenance Engine for Power Cables in Distribution Grids, Master Thesis, Federal Institute of Technology (ETH), 2-4 (2018)

Espinosa, F., A. Días y N. Back, Un Procedimiento de Evaluación de las Condiciones Necesarias para Innovar la Gestión de Mantenimiento en una Empresa, Doi: 10.4067/S0718-07642008000100012, Información Tecnológica 19(1), 97-104 (2008)

Giraldo, N.D, Capítulo 5: Validación del modelo de regresión, Notas de Clase series de tiempo con R, Universidad Nacional de Colombia, 80-82, Medellín, Colombia (2006)

Gogtay, N. y U. Thatte, Principles of Correlation Analysis, Journal of Association of Physicians of India, 65, 78-81 (2017)

Hanke, J. y D. Wichern, Chapter 3: Exploration of data patterns and introduction to response techniques, Business Forecasting, 9th Ed., Pearson, 65-68 USA (2010a)

Hanke, J. y D. Wichern, Chapter 4: Methods of moving and smoothing averages, Business Forecasting, 9th Ed., Pearson, 119-120 USA (2010b)

Hanke, J. y D. Wichern, Chapter 8: Regression with time series data, Business Forecasting, 9th Ed., Pearson, 336-343 USA (2010c)

Jaha Begum, K. y A. Ahmed, The Importance of Statistical Tools in Research Work, Journal of Scientific and Innovative Mathematical Research (IJSIMR), ISSN 2347-307X, 3(12), 50-58 (2015)

Krenek, J., K. Kuca y otros tres autores, Application of Artificial Neural Networks in Condition Based Predictive Maintenance, Doi: 10.1007/978-3-319-31277-4_7, Recent Developments in Intelligent Information and Database Systems, (2016)

Kolte, S. y U. Dabade, Machine Operational Availability Improvement by Implementing Effective Preventive Maintenance Strategies - A Review and Case Study, International Journal of Engineering Research and Technology, ISSN 0974-3154, 10(1), 700-708 (2017)

Kumar Pinjala, S., L. Pintelon y A. Vereecke, An empirical investigation on the relationship between business and maintenance strategies, Doi: 10.1016/j.ijpe.2004.12.024, International Journal of Production Economics, 104(1), 214-229 (2006)

Lee, T. y C. Park, Tests for serial correlation in mean and variance of a sequence of time series objects, Doi: 10.1080/00949655.2016.1217336, Journal of Statistical Computation and Simulation, 87(3), 478 -492 (2017) 
Liu, Q., X. Chen y otros 4 autores, A Novel Short-Medium Term Satellite Clock Error Prediction Algorithm Based on Modified Exponential Smoothing Method, Doi: 10.1155/2018/7486925, Mathematical Problems in Engineering, 3, 1-7 (2018)

Meimand, S., H. Granjalikhan Hakemi y H. Ismail, Demand Forecasting: A Review on Qualitative Methods and Surveyed Done By Delphi Technique, University Technology Malaysia, 3-4 (2012)

Morales, E, Introducción a la Econometría, Capítulo 11: Modelos de Selección Cualitativa, ABYA-YALA, Quito, Ecuador, $586(2001)$

Ostertagova, E. y O. Ostertag, Forecasting Using Simple Exponential Smoothing Method, Doi: 10.2478/v10198-012-00342, Acta Electrotechnica et Informatica, 12(3), 62-66 (2012)

Paithankar, A. y C. Snehamoy, Forecasting time-to-failure of machine using hybrid Neuro-genetic algorithm - a case study in mining machinery, Doi: 10.1080/17480930.2016.1262499, International Journal of Mining Reclamation and Environment, 32(3), 1-14 (2016)

Pamuk, N., The assessment of preventive maintenance activities in relationship with the electrical equipments of Northwestern Anatolian electricity power network, Doi: 10.16984/saufebbilder.25967, SAÜ Fen Bil Der, 19(1), 99-106 (2015)

Prasad, S. y P. Prasad, Deep Recurrent Neural Networks for TimeSeries Prediction, Cornell University, IEEE, (2014). Ravinder, H.V. Determining The Optimal Values Of Exponential Smoothing Constants - Does Solver Really Work? Montclair, Doi: 10.19030/ajbe.v9i1.9574, American Journal of Business Education, 6(3), 347-360 (2013)

Rao, B., R. Prasad y R. Kantham, Monitoring Software Reliability using Statistical Process Control: An Ordered Statistics Approach, International Journal of Computer Applications, ISSN: 0975 - 8887, 32(7), 28-33 (2011)

Sánchez, G., H. Pérez y M. Nakano, Red Neuronal Creciente Usando Perturbación Simultánea, Doi: 10.4067/S071807642004000500008, Información Tecnológica, 15(5), 45-52 (2004)

Sujata, B., A. Prashanth y P. Sainath, Machine Learning Based Predictive Maintenance Strategy: A Super Learning Approach with Deep Neural Network, Doi: 10.1109/WMED.2018.8360836, Workshop on Microelectronics and Electron Devices (WMED), (2018)

Tealab, A., Time Series Forecasting Using Neural Networks, https://doi.org/10.1016/j.fcij.2018.10.003, Future Computing and Informatics Journal, 3(2), 334-340 (2018)

Valtanen, P. y P. Perälä, Standard operating procedures, preventive maintenance and layered process audits, Savonia University of Applied Sciences, Tomado de: https://www.theseus.fi/bitstream/handle/10024/128796/ Valtanen_Petri.pdf.pdf?sequence=1\&isAllowed=y, 9-11 (2017)

Westholm, J., Event Detection and Predictive Maintenance using Component Echo State Networks, Master's Thesis in Mathematical Statistics, Tomado de: https://up.lub.lu.se/student-papers/search/publication/8931445, (2018) 